Original article

\title{
Role of bacterial agents in newborn babies with septicemia
}

\author{
Aliyar Pirouzi ${ }^{\mathrm{a}}$, Mohammad Javad Mohammadi ${ }^{\mathrm{b}}$, Ahmad Abdollahi ${ }^{\mathrm{a}}$, Hamideh Mohseni ${ }^{\mathrm{c}}$, \\ Elham Rezaee $^{\mathrm{a}}$, Sahar Geravandi ${ }^{\mathrm{d}}$, Hossein Foruozandeh ${ }^{\mathrm{a}, *}$ \\ ${ }^{a}$ Cellular and Molecular Research Center, Gerash University of Medical Sciences, Gerash, Iran \\ ${ }^{\mathrm{b}}$ Department of Environmental Health Engineering, School of Public Health AND Environmental Technologies Research Center, Ahvaz Jundishapur University of Medical \\ Sciences, Ahvaz, Iran \\ ${ }^{\mathrm{c}}$ Islamic Azad University of Larestan, Larestan, Iran \\ ${ }^{\mathrm{d}}$ Asadabad School of Medical Sciences, Asadabad, Iran
}

\section{A R T I C L E I N F O}

\section{Keywords:}

Septicemia

Newborn babies

Bacterial agents

Antibiotic resistance

Evaz

Iran

\begin{abstract}
A B S T R A C T
Introduction: This research is aimed to determine the most common bacterial agents in septicemia of newborn babies. The blood infection is a clinical syndrome caused by invading microorganisms or their toxins into the blood.

Objective: This study was conducted in Abd al-Hamid Omidvar Hospital, Evaz, Iran, on 516 newborn babies and infants with an average age of eight months suspected to infection.

Method: Followed by collecting of blood samples and performing of microbial culture, evaluation of antibiotic sensitivity was performed by disc diffusion method on agar medium.

Results: Among 516 samples of blood cultures, 20 samples (3.8\%) of cultures were positive, of which 12 cases (3.9\%) were boy and 8 cases $(3.7 \%)$ were girl. Bacteria which cause blood infections include Staphylococcus epidermidis (30\%), Klebsiella pneumonia (30\%), Escherichia coli (25\%), Enterobacter (10\%) and Staphylococcus aureus (5\%). The most sensitivity Gram positive and Gram negative strains were both to ciprofloxacin and the highest resistance of Gram negative strains was against cefixime and cephalexin, and the highest resistance of Gram positive strains was against tetracycline and oxacillin.

Conclusion: paying attention to the prevalence of infection with Staphylococcus epidermidis in this study and similar studies, with correct use of antibiotics for treatment and observation of hygiene principles in pediatrics and neonatal wards can reduce mortality and morbidity caused by the disease.
\end{abstract}

\section{Introduction}

Blood is one of the body fluids, which is normally sterile, but this sterility may be altered under a series of conditions and microorganisms can enter the blood stream through an infectious process and cause septicemia. ${ }^{1}$ The blood infection is a clinical syndrome caused by invading microorganisms or their toxins into the blood stream and includes bacteremia, septicemia, virmeia and/or parasitemia. ${ }^{2}$ Septicemia or sepsis is a systematic inflammatory response syndrome and in fact is the same bacteremia plus clinical protests, bacterial invasion signs into the blood and toxin production by microorganisms which, if it is left unknown and untreated, it can quickly progress to severe sepsis, septic shock and death. ${ }^{3}$ One of the main diseases that occur as a result of the complications of blood infection is defined Sepsis in neonatal. So, early diagnosis of microorganisms in the blood and selection of appropriate antibiotics causes a reduction in morbidity and mortality. ${ }^{4}$ The blood infection in newborn babies is a clinical syndrome and is referred to a series of systematic signs in less than 28 days old infants with positive blood culture. These infections are divided into two groups: early (created during the early $72 \mathrm{~h}$ of life are probably related to risk factors of fetal period) and late groups (appeared after the first $72 \mathrm{~h}$ and often caused by hospital-acquired infections). ${ }^{5}$ Various factors including genetic, social, health and nutritional differences and environmental conditions are effective on bacterial prevalence and causal factors of these infections. ${ }^{6-10}$ The incidence of neonatal infections varies from a country to another and from a county to another and even varies in a county at different times. ${ }^{6}$ The difference is likely related to the amount of premature birth, prenatal care, and quality of childbirth and position of neonatal care. ${ }^{11,12}$ Given to the high importance of infection in newborns and given that the most of actual infections caused by

\footnotetext{
* Corresponding author. Cellular and Molecular Research Center, Gerash University of Medical Sciences, Gerash, Iran. Tel.: +989382109998; fax: 00987152424033.

E-mail address: hosainforuozandeh@yahoo.com (H. Foruozandeh).
} 
organisms are thought contamination by mistake and simply are ignored as well as given that the reduction of mortality in neonatal period is one of the most important goals of health and hygiene programs in Iran, so performance of this study and determination of risk factors for infection and its prevention as well as early diagnosis of the blood infection can be an effective help to treat the infection and to reduce mortality caused by it.

Determination of bacterial agents in newborn babies' septicemia and identification of drug resistance in Abd al-Hamid Omidvar Hospital, Evaz, Iran.

\section{Methods}

This is a descriptive-analytic study and was performed on 516 newborns and infants who hospitalized in Abd al-Hamid Omidvar Hospital, Evaz, Iran, from 22 June 2015 to 26 October 2016. Blood samples were obtained from those newborns and infants suspected to septicemia. The preparation of blood samplings performed properly according to prescription of physician and by three different persons and in three different times in related ward and they sent to laboratory (the blood samples in this Hospital were sent to laboratory once). The amount of $2-5 \mathrm{cc}$ of venous blood of each babies were poured into trypton soy broth (TSB) or gelatin peptone culture media containing anticoagulant sodium polyanethol sulfonate (SPS) and sent to the laboratory. This culture media is incubated for $24 \mathrm{~h}$ and then at the times of 24,48 and $72 \mathrm{~h}$ and 10 days after that time sub-culture were prepared on MacConkey agar (or EMB), blood agar, chocolate agar and thioglycollate broth culture media. Gram-negative and/or gram positive type of bacteria was recognized according to culture on EMB and blood agar media and on this base differential tests were done. Catalase, coagulase, novobiocin and bacitracin tests was conducted as differential tests for Gram positives, and the differential tests for Gram negatives was Gallery (collection of sodium citrate, TSI, urea, SIM and chrome agar) in this center. Sensitivity test to antibiotics was performed by disc diffusion method for all the positive tests. The intended colonies on Mueller Hinton medium were cultured to a thickness of $4 \mathrm{~mm}$ and disc dispensing was performed by disc diffusion method. The antibiotics discs used in this method include:

Cefotaxime, ceftazidime, ceftriaxone, cephalexin, chloramphenicol, cinoxacin, ciprofloxacin, gentamicin, imipenem, nalidixic acid, nitrofurantoin, oxacillin, penicillin, sulfamethoxazole, erythromycin, cefixime, tetracycline, ampicillin, vancomycin, amikacin, and rifampin.

\section{Results}

Of 516 newborns and infants suspected to septicemia and tested in this study, 304 cases (58.91\%) were boy and 212 cases (41.08\%) were girl that of them 20 cases $(3.8 \%)$ had positive blood culture. These babies were included 12 patient boys (3.94\%) and 8 patient girls (3.77\%).

Bacteria which cause blood infections include Staphylococcus epidermidis (30\%), Klebsiella pneumonia (30\%), Escherichia coli (25\%), Enterobacter (10\%) and Staphylococcus aureus (5\%). The most sensitivity of Gram positive and Gram negative strains were both to ciprofloxacin and the highest resistance of Gram negative strains was against cefixime and cephalexin, and the highest resistance of Gram positive strains was against tetracycline and oxacillin. The results summarize in Tables 1-4.

\section{Discussion}

Life expectancy of neonates is increasing with the help of advances in neonatology, new life support techniques, and new treatment modalities. As a result of increased life expectancy, duration of hospitalization is also increasing, and in spite of new antibiotics and new supportive measures, sepsis and its treatment has become a major problem in the Neonatal Intensive Care Unit (NICU). ${ }^{13}$ Therefore, by considering
Table 1

Type and number of bacterial isolates in neonates with sepsis.

\begin{tabular}{lll}
\multicolumn{2}{l}{ Bacteria that made the blood culture positive } & \\
Bacterium & The number & Percentage (\%) \\
\hline Staphylococcus epidermidis & 6 & 30 \\
Klebsiella pneumonia & 6 & 30 \\
Escherichia coli & 5 & 25 \\
Enterobacter & 2 & 10 \\
Staphylococcus aureus & 1 & 5 \\
\hline
\end{tabular}

Table 2

The results of blood cultures according to gender.

The results obtained from blood cultures according to gender

\begin{tabular}{lllll}
\hline \multicolumn{2}{l}{ Positive Culture } & \multicolumn{2}{l}{ Negative Culture } \\
\hline Sex & Number & Percentage (\%) & Number & Percentage (\%) \\
\hline Girl & 8 & 3.77 & 204 & 96.23 \\
Boy & 12 & 3.94 & 292 & 96.06 \\
\hline
\end{tabular}

Table 3

Antimicrobial susceptibility pattern of gram positive isolated bacteria to commonly used antibiotics in present study.

Gram Positive Antibiogram Table

\begin{tabular}{lllll}
\hline \multicolumn{1}{l}{ Sensitivity } & & \multicolumn{3}{c}{ Resistance } \\
\hline Antibiotics & Number & Percentage (\%) & Number & Percentage (\%) \\
\hline Ciprofloxacin & 6 & 85.7 & - & - \\
Erythromycin & 4 & 57.1 & 3 & 42.8 \\
Gentamicin & 4 & 57.1 & 1 & 14.2 \\
Sulfometoxasole & 4 & 57.1 & 1 & 14.2 \\
Oxacillin & 3 & 42.8 & 3 & 42.8 \\
Cephalexin & 3 & 42.8 & - & - \\
Penicillin & 2 & 28.5 & 3 & 42.8 \\
Cinoxacin & 1 & 14.2 & - & - \\
Tetracycline & - & - & 3 & 42.8 \\
Cefixime & - & - & 1 & 14.2 \\
Ampicillin & - & - & 1 & 14.2
\end{tabular}

Table 4

Antimicrobial susceptibility pattern of gram negative isolated bacteria to commonly used antibiotics in present study.

Gram Negative Antibiogram Table

\begin{tabular}{|c|c|c|c|c|}
\hline \multicolumn{3}{|l|}{ Sensitivity } & \multicolumn{2}{|c|}{ Resistance } \\
\hline Antibiotics & Number & Percentage (\%) & Number & Percentage (\%) \\
\hline Ciprofloxacin & 13 & 100 & - & - \\
\hline Gentamicin & 11 & 84.6 & 2 & 15.3 \\
\hline Sulfamethoxazole & 8 & 47.05 & 1 & 7.6 \\
\hline Amikacin & 7 & 53.8 & 3 & 23.07 \\
\hline Ceftazidime & 7 & 53.8 & - & - \\
\hline Cefixime & 6 & 46.1 & 6 & 46.1 \\
\hline Ceftriaxone & 5 & 38.4 & 4 & 30.7 \\
\hline Cefotaxime & 5 & 38.4 & 3 & 23.07 \\
\hline Cephalexin & 4 & 30.7 & 6 & 46.1 \\
\hline Imipenem & 3 & 23.07 & - & - \\
\hline Chloramphenicol & 2 & 15.3 & - & - \\
\hline Nalidixic acid & 1 & 7.6 & - & - \\
\hline Nitrofurantoin & - & - & 4 & 30.7 \\
\hline Cinoxacin & - & - & 2 & 15.3 \\
\hline
\end{tabular}

the importance of blood infection in newborn babies as the most common cause of death, this study was conducted to determine the role of bacterial agents of septicemia in newborn babies as well as to 
identify the level of antibiotic resistance against isolated bacteria from the positive cultures. The results of present study showed that the most common bacteria that produced septicemia were Staphylococcus epidermidis and Klebsiella pneumonia. Gram positive bacteria had the highest sensitivity to ciprofloxacin and were somewhat resistant against erythromycin, tetracycline, oxacillin and penicillin. The highest sensitivity in the case of Gram negative bacteria was also allocated to ciprofloxacin and cefixime; and cephalexin are accounted as resistant antibiotics for Gram negatives. Other researcher also were investigated the pattern of septicemia in newborn babies, causative agent and their antibiotic resistance. According to a study that was conducted on children hospitalized in Tabriz medical center, Iran, coagulase-negative Staphylococci and Klebsiella pneumonia were reported as the most common bacteria available in the blood culture of newborn babies. ${ }^{14}$ According to the results of investigation of newborn babies hospitalized in Shahid Arefian Hospital, Urumiah, Iran, the most available bacteria were included coagulase-negative Staphylococci and Klebsiella pneumonia. The highest sensitivity of Gram negative bacteria in the above study was to ciprofloxacin and the highest resistance was against cefotaxime. However, Gram negative bacteria in the current study did not show superior resistance against cefotaxime. Gram positive bacteria in the investigation performed in Shahid Arefian Hospital, Urumiah, had become resistant to ampicillin and sensitive to vancomycin and ceftriaxone. ${ }^{15}$ In 1980s, Staphylococcus aureus and Staphylococcus epidermidis have been identified as the main cause of infection in the neonatal intensive care unit. ${ }^{16}$ Based on results obtained from a study was performed in Mazandaran in 2003-2004, coagulase-negative Staphylococcus as the most common disease-causing strains in newborns was isolated especially in the use of venous catheters. ${ }^{17}$ These bacteria have been saprophytes in environment and have been considered as a part of normal flora of the skin; and the probability of infection in newborns in the first week of life is through passing of bacteria from the birth canal, contacting with air and personnel of hospital.

\section{Conclusion}

According to this study and similar studies conducted in this regard, an increase in antibiotic resistance is observed in pathogenic strains. So by adopting an appropriate strategy for exact isolation of pathogenic agents and proper use of antibiogram in treatment plan of newborns can reduce mortality and complications of bacterial infections in them and duration of hospitalization and related costs will also reduce in those patients.

\section{Financial disclosure}

We have no financial interests related to the material in the manuscript.

\section{Funding/support}

This work was financially supported by grant: (975) from Gerash University of Medical Sciences, Iran.

\section{Declaration of competing interest}

Authors have no conflict of interests.

\section{Acknowledgments}

The authors would like to extend their gratitude to the medical staff of Abd al-Hamid Omidvar Hospital of Evaz and all patients who cooperated with us in this study.

\section{References}

1. Tallur SS, Kasturi A, Nadgir SD, Krishna B. Clinico-bacteriological study of neonatal septicemia in Hubli. Indian J Pediatr. 2000;67:169-174.

2. Hall MJ, Williams SN, DeFrances CJ, Golosinskiy A. Inpatient care for septicemia or sepsis: a challenge for patients and hospitals. NCHS Data Brief. 2011;62:1-7.

3. Fanaroff AA, Korones SB, Wright LL, et al. Incidence, presenting features, risk factors and significance of late onset septicemia in very low birth weight infants. Pediatr Infect Dis J. 1998;17:593-598.

4. ERIKSSON M. Neonatal septicemia. Acta Paediatr. 1983;72:1-8.

5. Coventry K, ISBISTER C. A bacteriological and clinical study of infection in newborn babies in a maternity hospital nursery. Med J Aust. 1951;2:394-396.

6. Zaidi AK, Huskins WC, Thaver D, Bhutta ZA, Abbas Z, Goldmann DA. Hospital-acquired neonatal infections in developing countries. The Lancet. 2005;365:1175-1188.

7. Saeidimehr S, Geravandi S, Rahim F, et al. Nosocomial infection rates during one year in naft grand hospital, Ahvaz, Iran. Jundishapur J Health Sci. 2015;7.

8. Nashibi R, Afzalzadeh S, Mohammadi MJ, Yari AR, Yousefi F. Epidemiology and treatment outcome of mucormycosis in Khuzestan, Southwest of Iran. Arch Clin Infect Dis. 2017;12:e37221.

9. Nashibi R, Mohammadi MJ, Alavi SM, et al. Infection after open heart surgery in Golestan teaching hospital of Ahvaz, Iran. Data Brief. 2018;16:478-482.

10. Geravandi S, Soltani F, Mohammadi MJ, et al. The effect of education on the nursing care quality of patients who are under mechanical ventilation in ICU ward. Data Brief. 2018;16:822-827.

11. Stoll BJ, Hansen N, Fanaroff AA, et al. Late-onset sepsis in very low birth weight neonates: the experience of the NICHD Neonatal Research Network. Pediatrics. 2002;110:285-291.

12. Makhoul IR, Sujov P, Smolkin T, Lusky A, Reichman B. Epidemiological, clinical, and microbiological characteristics of late-onset sepsis among very low birth weight infants in Israel: a national survey. Pediatrics. 2002;109:34-39.

13. Yalaz M, Çetin H, Akisu M, Aydemir S, Tunger A, Kultursay N. Neonatal nosocomial sepsis in a level-III NICU: evaluation of the causative agents and antimicrobial susceptibilities. Turk J Pediatr. 2006;48:13-19.

14. Ghotaslou R, Ghorashi Z, Nahaei M. Klebsiella pneumoniae in neonatal sepsis: a 3year-study in the pediatric hospital of Tabriz Iran. Jpn J Infect Dis. 2007;60:126.

15. Sayehmiri K, Nikpay S, Azami M, Pakzad I, Borji M. The prevalence of neonatal septicemia in Iran: a systematic review and meta-analysis study. J Shahrekord Univ Med Sci. 2017;19.

16. Villari P, Sarnataro C, Iacuzio L. Molecular epidemiology of Staphylococcus epidermidis in a neonatal intensive care unit over a three-year period. J Clin Microbiol. 2000;38:1740-1746.

17. Behzadnia S, Davoudi A, Rezai MS, Ahangarkani F. Nosocomial infections in pediatric population and antibiotic resistance of the causative organisms in north of Iran. Iran Red Crescent Med J. 2014;16. 